\title{
Provisional Prosthetic Management of Mobile Teeth in Conjunction with a Removable Partial Denture Using Orthodontic Wire
}

\author{
Won-suk Oh, DDS, $\mathrm{MS}^{1}$ \& Berna Saglik, DDS ${ }^{2}$ \\ ${ }^{1}$ Clinical Associate Professor, Department of Biologic \& Materials Sciences, Division of Prosthodontics, University of Michigan School of Dentistry, \\ Ann Arbor, MI \\ ${ }^{2}$ Clinical Assistant Professor, Department of Biologic \& Materials Sciences, Division of Prosthodontics, University of Michigan School of Dentistry, \\ Ann Arbor, MI
}

\author{
Keywords \\ Precision attachment-retained removable \\ partial dentures; provisional prosthetic \\ management; abutment tooth mobility; \\ support; stability.

\section{Correspondence} \\ Won-suk Oh, Department of Biologic and \\ Materials Sciences, Division of \\ Prosthodontics, University of Michigan \\ School of Dentistry, $1011 \mathrm{~N}$. University, \\ Rm K1014A, Ann Arbor, MI 48109-1078. \\ E-mail: ohws@umich.edu
}

Accepted September 8, 2008

doi: 10.1111/j.1532-849X.2009.00494.x

\begin{abstract}
Precision attachment-retained removable partial dentures eliminate the use of visible clasps and improve the esthetic appearance of the smile; however, terminal abutment teeth may be subject to unfavorable stresses under function when misused. A provisional prosthetic management technique that incorporates an orthodontic wire to assist cross-arch support and stability of the periodontally weakened abutment teeth is described. This technique is simple, reversible, does not alter the esthetic appearance of the smile, and controls the mobility of the abutment teeth until a definitive treatment plan is established.
\end{abstract}

Tooth mobility has been associated with the use of removable partial dentures (RPDs), in particular, distal-extension RPDs, due to the differences in the compressibility of the tooth and mucosa (Fig 1A). ${ }^{1-3}$ Abutment teeth are subjected to compressive, tensile, and torsional stresses under masticatory function and at rest. ${ }^{4,5}$ An RPD framework is designed to distribute the stresses across the arch and maximize the support and stability by employing multiple rests on the remaining teeth and extending denture bases on the residual ridges. ${ }^{5,6}$

Abutment teeth are often connected to allow single-rooted teeth to have the increased stability of multirooted teeth, which shifts the center of rotation to the interdental septum and reduces the degree of turning moment. ${ }^{7,8}$ In an in vitro analysis, stresses on double abutment teeth were found to be reduced by $35 \%$ as compared to single abutment teeth. ${ }^{8}$ This can be an important consideration with regard to periodontally weakened abutment teeth and the construction of intracoronal precision attachment-retained distal-extension RPDs (PARPDs), which may exert more stress on the abutment teeth than conventional clasp design. ${ }^{9,10}$

This article describes a technique for the provisional management of the periodontally weakened abutment teeth in conjunction with a maxillary PARPD prosthesis. A stainless steel, round $0.032^{\prime \prime}(0.81 \mathrm{~mm})$ orthodontic wire was contoured against the labial surfaces of the remaining dentition across the arch and attached to the existing PARPD prosthesis to consolidate the teeth as one unit and provide cross-arch support and stability to the mobile abutment teeth. This procedure is simple and reversible, benefits the patient without altering the esthetic appearance of the smile, and allows for controlling and monitoring the support and stability of the mobile abutment teeth until a definitive treatment plan is established.

\section{Technique}

(1) Make an irreversible hydrocolloid impression (Jeltrate, Dentsply Caulk, Milford, DE) over the existing maxillary PARPD prosthesis intraorally using a metal stock tray (GC America Inc, Alsip, IL).

(2) Coat the intaglio surface of the prosthesis with a thin layer of petroleum jelly (Unilever, Greenwich, CT) and block out the undercuts in the prosthesis using wet pumice (Whip Mix Corp, Louisville, KY). Pour the impression in fast-setting plaster (Mounting Plaster, Whip Mix Corp) to relate the prosthesis to the cast. 
A

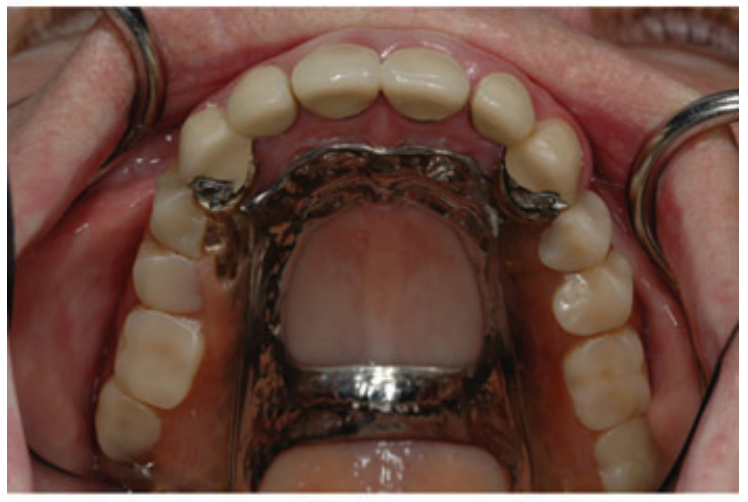

B



C

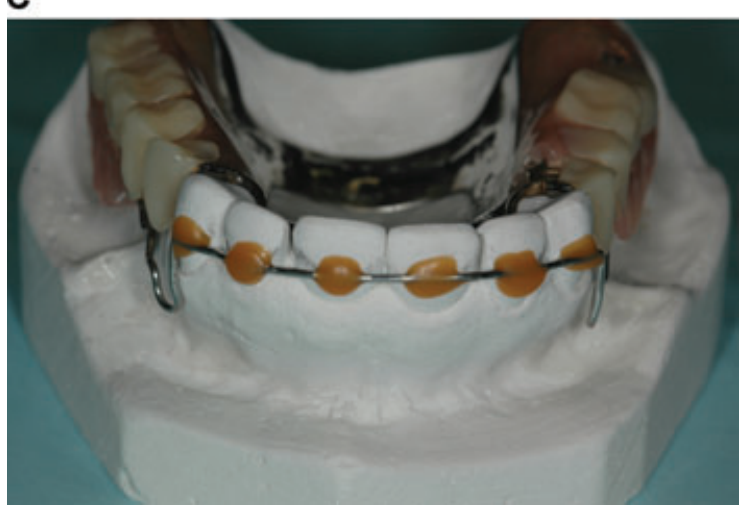

Figure 1 (A) Intraoral occlusal view of the maxillary PARPD prosthesis. (B) Occlusal view of the PARPD prosthesis on the cast. Note a $0.032^{\prime \prime}$ thick orthodontic wire contoured and secured on the plaster teeth. (C) Frontal view of the cast. Note the position of the wire at the height of contour of the teeth.

(3) Place the cast on the surveyor table (J.M. Ney Co, Hartford, CT), and tilt the table to match the path of insertion of the existing PARPD prosthesis as reference to the attachment. Draw the survey line on the labial surfaces of the teeth to indicate the height of contour, and separate the prosthesis from the cast.

(4) Prepare the existing PARPD prosthesis to receive a wire within the acrylic base using tungsten carbide burs

\section{A}

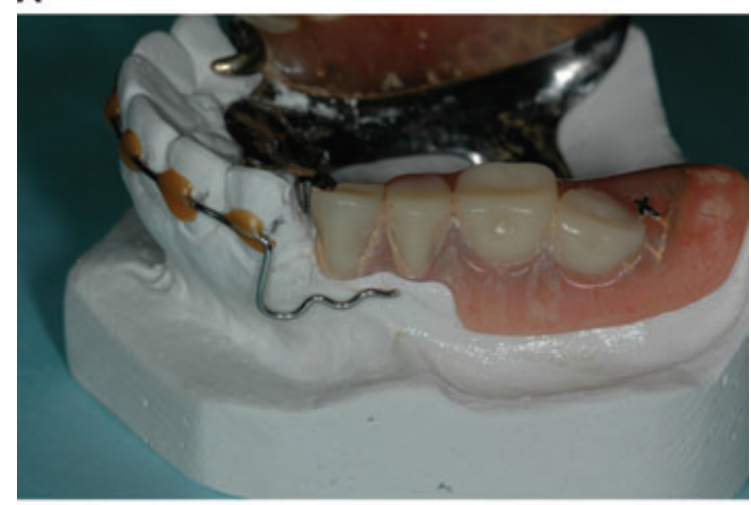

B

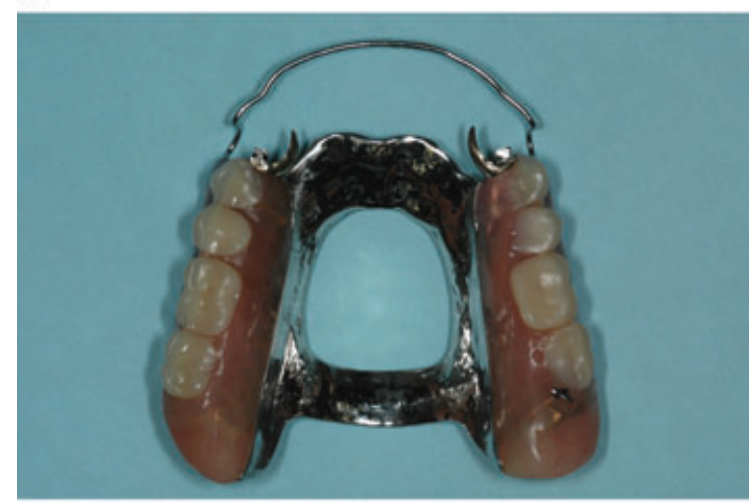

C

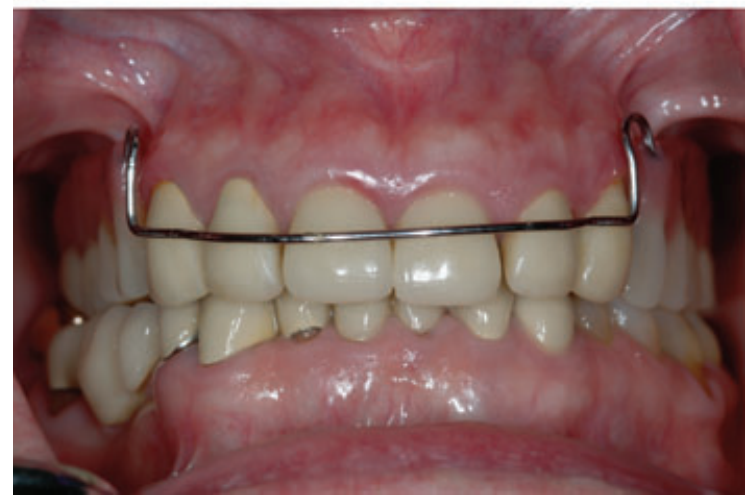

Figure 2 (A) Lateral view of the PARPD prosthesis on the cast. Note the multiple bends at the end of the wire to aid mechanical retention with acrylic resin. (B) Occlusal view of the modified maxillary PARPD. (C) Frontal view of the maxillary teeth. Note the round wire across the teeth without altering the esthetic appearance of the smile.

(Brasseler USA Inc, Savannah, GA) and reseat the prosthesis on the cast.

(5) Contour a stainless steel, round orthodontic wire $\left(0.032^{\prime \prime}\right.$ Standard Round Wire, 3M Unitek Corp, Monrovia, CA) to adapt to the labial surfaces of the remaining teeth at the height of contour using Bird Beak pliers (3M Unitek Corp) (Fig 1B, C). Create multiple bends at the end of the wire to ensure mechanical locking within the acrylic 
resin of the denture base (Fig 2A). Note the U-shaped loop on each side of the wire crossing the gingiva at a right angle, permitting adjustments when needed. Secure the contoured wire in place on the cast using sticky wax (Kerr USA, Romulus, MI).

(6) Add increments of autopolymerizing pink acrylic resin (Jet Denture Repair Acrylic, Dental Mfg Co Inc, Wheeling, IL) using a sable brush to attach the wire to the existing denture base. Return the denture prosthesis to a pressure pot (AcriDense V Pneumatic Curing Unit, GC America Inc) with $100^{\circ} \mathrm{F}$ water at $25 \mathrm{psi}$ for at least 10 minutes.

(7) Separate the repaired and modified denture prosthesis from the cast after curing, trim the excess, and polish (Fig 2B). Fit the modified PARPD prosthesis in the mouth using pressure indicating paste (Mizzy Inc, Cherry Hill, $\mathrm{NJ}$ ), check the occlusal contacts, and reinsert the prosthesis in the mouth (Fig 2C).

(8) Provide home-care instructions, make recall appointments to control and monitor the abutment tooth mobility, and plan for definitive treatment.

\section{Summary}

A provisional prosthetic management technique has been presented to provide support and stability to the periodontally weakened abutment teeth in conjunction with a maxillary PARPD prosthesis. A stainless steel orthodontic wire is contoured against the labial surfaces of the entire remaining dentition and attached to the existing PARPD prosthesis to consolidate the teeth as one unit and assist support and stability of the abutment teeth until a definitive treatment plan is established. Potential risk of lateral stresses is minimized by placing the wire at the height of contour of the teeth. The wire is displaced incisally without torquing the abutment teeth under function. This technique is simple and reversible, permits the esthetic appearance of the smile, and controls the mobility of the abutment teeth until the establishment of a definitive treatment plan.

\section{References}

1. Goodkind RJ: The effects of removable partial dentures on abutment tooth mobility: a clinical study. J Prosthet Dent 1973;30:139-146

2. Kern M, Wagner B: Periodontal findings in patients 10 years after insertion of removable partial dentures. J Oral Rehabil 2001;28:991-997

3. Akaltan F, Kaynak D: An evaluation of the effects of two distal extension removable partial denture designs on tooth stabilization and periodontal health. J Oral Rehabil 2005;32:823-829

4. Clayton JA, Jaslow CA: Measurement of clasp forces on teeth. J Prosthet Dent 1971;25:21-42

5. Frechette AR: Partial denture planning with special reference to stress distribution. J Prosthet Dent 1951;1:710-724

6. Kratochvil FJ: Maintaining supporting structures with a removable partial prosthesis. J Prosthet Dent 1971;25:167-174

7. Talbot TR: Review of the Swinglock removable partial denture. Int J Prosthodont 1991;4:80-88

8. Aydinlik E, Dayangaç B, Celik E: Effect of splinting on abutment tooth movement. J Prosthet Dent 1983;49:477-480

9. Chou TM, Caputo AA, Moore DJ, Xiao B: Photoelastic analysis and comparison of force transmission characteristics of intracoronal attachments with clasp distal extension removable partial dentures. J Prosthet Dent 1989;62:313-319

10. Chou TM, Eick JD, Moore DJ, Tira DE: Steriophotogrammetric analysis of abutment tooth movement in distal extension removable partial dentures with intracoronal attachments and clasps. J Prosthet Dent 1991;66:343-349 